\title{
Diagnostic Value of a Multidisciplinary Clinic for Intellectual Disability
}

\author{
Clara van Karnebeek, Tyler Murphy, Wynona Giannasi, Marion Thomas, \\ Mary Connolly, Sylvia Stockler-Ipsiroglu
}

\begin{abstract}
Background: Identification of the underlying cause of intellectual disability (ID) is important as it improves genetic counselling, management, adaptation; yet its etiologic heterogeneity is challenging and often leads to an expensive work-up. Methods: To improve this diagnostic trajectory, the multidisciplinary Complex Diagnostic Clinic (CDC) was established for ID patients with unexplained complex systemic and/or neurologic features that were referred to the CDC and evaluated by three medical specialists followed by multi-disciplinary rounds. Analyses included surveys and interviews, (retrospective) chart review, costs calculations and comparison. Results: 24 children (9 male) were evaluated during seven clinics held over 16 months. The average patient age was 7 years 11 months (range 9 months-18 years). All the children had previously been seen by 2-10 specialist services. The diagnostic yield of the CDC was higher than expected with confirmed and working diagnoses in 11 (46\%) and 9 (38\%) children respectively. Cost-savings included fewer trips to hospital and fewer tests via more streamlined evaluations. Positive feedback was received from both families and medical professionals. Conclusions: The CDC represents an innovative model of personalized care. Specialist collaboration in the interpretation of relevant clinical, biochemical and genomic data resulted in diagnoses, where none had previously been possible.
\end{abstract}

RÉSUMÉ: Valeur diagnostique d'une clinique multidisciplinaire pour la déficience intellectuelle. Contexte : L'identification de la cause de la déficience intellectuelle (DI) est importante parce qu'elle permet d'améliorer le conseil génétique, le traitement et l'adaptation du patient. Cependant, l'hétérogénéité de la DI explique que son diagnostic demeure un défi et donne souvent lieu à des examens coûteux. Méthode : La clinique multidisciplinaire pour le diagnostic de maladies complexes (CMDMC) a été établie afin d'améliorer le parcours diagnostique pour les patients atteints de DI qui ont des caractéristiques systémiques et/ou neurologiques complexes. Les patients référés à la CMDMC ont été évalués par trois spécialistes puis par une équipe multidisciplinaire. L'évaluation des patients inclut des enquêtes et des entrevues, une revue de dossier rétrospective, des calculs et des comparaisons de coûts. Résultats : 24 enfants, dont 9 étaient des garçons, ont été évalués lors de 7 cliniques tenues au cours d'une période de 16 mois. L'âge moyen des patients était de 7 ans et 11 mois (écart de 9 mois à 18 ans). Tous les enfants avaient été évalués dans le passé par 2 à 10 services spécialisés. Le taux de diagnostic de la CMDMC a été plus élevé que prévu, soit un diagnostic confirmé chez 11 enfants (46\%) et un diagnostic provisoire chez 9 enfants (38\%). Une diminution du nombre de voyages pour se rendre à l'hôpital et de tests parce que les évaluations étaient simplifiées a permis de réaliser des économies. Nous avons reçu des commentaires favorables de la part des familles et des professionnels de la santé. Conclusions : La CMDMC constitue un modèle innovateur de soins personnalisés. La collaboration des spécialistes dans l'interprétation de données cliniques, biochimiques et génomiques pertinentes a permis de poser des diagnostics alors qu'il n'avait pas été possible de le faire antérieurement.

Can J Neurol Sci. 2014; 41: 333-345

Intellectual Disability (ID) affects $2.5 \%$ of the population worldwide. This life-long disability manifests before the age of 18 years, and is characterized by significant limitations in intellectual functioning (IQ $<70)$ as well as in adaptive behavior, which comprises conceptual, social and practical skills ${ }^{1}$. If formal testing is unavailable, school report cards, direct clinical observation and reports of third party informants are used to determine the presence of ID. In children under five years-ofage, IQ tests are not deemed reliable, and the term 'global developmental delay' is used, defined by significant limitations of two or more developmental domains (e.g. fine/gross motor skills, speech/language, cognition, social/personal, activities of daily living), i.e. a performance of two standard deviations or more below the mean on age-appropriate, standardized, normreferenced testing ${ }^{2}$. Here we apply ID as collective term for both. Affecting all areas of a patient's life, ID results in considerable psychological, social and economic burdens for patients, families and society as a whole ${ }^{3}$.

The etiology of ID is extremely diverse, including environmental factors (e.g. fetal substance exposure, peri- and postnatal brain injury), chromosomal abnormalities (e.g. Down syndrome) and single gene disorders (e.g. fragile X syndrome).

From the Division of Pediatric Neurology (TM, MC), Howe Consulting Group (WG), Department of Medical Genetics (MT), Division of Biochemical Diseases (SS, CvK), TIDE-BC, B.C. Children's Hospital, University of British Columbia, B.C., Canada. Received October 29, 2013. Final Revisions Submitted December 13, 2013 Correspondence to: Clara D.M. van Karnebeek, University of British Columbia, Centre for Molecular Medicine and Therapeutics, TIDE-BC, Division of Biochemical Diseases, Department of Pediatrics, B.C. Children's Hospital, Room K3-201, 4480 Oak Street, Vancouver B.C. V6H 3V4, Canada. Email: cvankarnebeek@cw.bc.ca. 
Consequently, the clinical presentation of ID in the majority of patients is multi-faceted and includes one or more of the following symptoms: epilepsy and other neurologic impairments; autism and behavioural/psychiatric disturbances; sleep problems; vision/hearing deficits; feeding challenges; dysmorphisms; and congenital organ abnormalities (anatomical and/or functional). Etiological heterogeneity makes establishing the diagnosis a challenge, often requiring many different tests by a variety of specialists. Genetic variants constitute the most frequent cause of ID and are demonstrable in 50\% or more of affected individuals ${ }^{4}$. Although considerable diagnostic advances have been made in recent years, including technologies that enable the identification of chromosomal copy number variants $(\mathrm{CNVs})$ through chromosome microarray analysis $(\mathrm{CMA})^{5}$, and single nucleotide mutations through next generation sequencing ${ }^{6}$, the etiological diagnosis in the remaining 40-50\% of individuals remains unknown 7 .

Establishing an etiologic diagnosis is important for several reasons. It offers both the opportunity to refine genetic counselling regarding recurrence risk and the possibility of early identification of other family members. A specific molecular defect (i.e. mutation, $\mathrm{CNV}$ ) allows for carrier testing, prenatal testing and/or pre-implantation testing. Furthermore, a diagnosis provides valuable information that the family can use to address their needs going forward; for example, access to services in the community can be facilitated, and many family support groups are organized around specific (groups of) diagnoses. Validation was found to be another important value attributed to a diagnosis, i.e. an explanation for a child's behaviour and appearance ${ }^{8}$. In addition, there is a potential greater benefit to the general population, as a specific diagnosis may provide clarification on the natural history of complex conditions. Importantly, prognosis can be more clearly defined, and management can be personally stratified and optimized, and the impact on the healthcare system reduced. Given the increasing number of genetic disorders for which a causal treatmenttargeting the pathophysiology - is available (81 inborn errors of metabolism in $2012^{9}$ ), early and accurate identification of the diagnosis underlying ID is of essence.

The Treatable Intellectual Disability Endeavour (TIDE-BC) (www.tidebc.org) merges basic research and care in a project funded as the first 'Collaborative Area of Innovation' at British Columbia's Children's Hospital (BCCH) in Vancouver, Canada. TIDE-BC is translating new knowledge into better health outcomes, utilizing state of the art and innovative technologies to improve diagnosis, treatment and care for children living with ID. All patients referred to BCCH with ID of unknown cause are evaluated using the two-tiered TIDE protocol (Supplementary Figure S1), which prioritizes the identification of treatable IDs. The protocol is supported by the Treatable ID App, available both as digital (www.treatable-id.org) and native too $1^{10}$. Patients are further evaluated according to international guidelines and practice parameters ${ }^{11}$, which advise genomic analyses, thyroid function and lead screening, audiology and vision testing all at low threshold; and neuro-imaging, neurophysiology, and molecular analyses on an individual basis.

Still, the challenges of establishing an etiologic diagnosis for ID patients are many, as the etio-pathogenesis encompasses so many different (rare) entities, which motivates the attending physician to initiate a wide-ranging diagnostic evaluation ${ }^{12}$. In our tertiary care centre, a chart review showed that this is especially true for ID patients with complex symptomatology affecting different organ systems. This expensive work-up may start with duplicate referrals by the community physician. Between 2007 and 2010, approximately 2000 new referrals for children with ID were made to Medical Genetics, Pediatric Neurology and/or Biochemical Diseases; $25 \%$ were seen by two of these services and $5 \%$ by all three services for a first opinion in parallel. Due to disjointed channels of communication, various specialists are often unaware of different specialities' simultaneous involvement and tests are ordered either in duplicate or not at all. An additional challenge is securing provincial funding for molecular and biochemical tests that are available only in laboratories outside of the province. The B.C. Ministry of Health Medical Services Plan approves funding requests only if the "outcome of the test leads to a therapeutic intervention"; thus, improved care via diagnostic confirmation and accurate genetic counselling are deemed insufficient. Involved specialists of the various services at $\mathrm{BCCH}$ felt that this situation often impedes a time-efficient and successful assessment of patients presenting with complex phenotypes, with inherent challenges in other areas including increased healthcare costs, long wait-times, and the uncertainties and practicalities of repeated travel and time off-work and school for the patient and his family. The need for a more coordinated approach was expressed repeatedly during monthly faculty meetings, as well as by families with a complex ID child during clinic visits.

\section{Methods}

\section{Complex Diagnostic Clinic (CDC) Aims}

We hypothesized that many of the previously mentioned challenges can be overcome by providing specialists with a platform for evaluating and discussing a given patient at one time and place-a multidisciplinary diagnostic clinic. Historically, such clinics focus on high-level care and management for patients with complex conditions such as amyotrophic lateral sclerosis, epilepsy and Down's syndrome $^{13-15}$. Examples at $\mathrm{BCCH}$ include the Spina Bifida Clinic and the Pain Clinic. However, multi-disciplinary diagnostic clinics are less frequent, with few reports in literature ${ }^{16}$.

Thus, the multidisciplinary CDC was established as part of the TIDE project, to improve the yield and the quality of diagnostic care for patients with complex ID. Here we report the quantitative and qualitative results of all 24 consecutive patients seen in our newly established CDC, held seven times during a pilot phase of 16 months (2011-2013), and compare this data to each patient's evaluation previous to the CDC, and to reports in the literature of comparable patient groups.

\section{CDC structure and organization}

The CDC Management Team includes a medical director (CvK, pediatrician-biochemical geneticist), clinic manager (MT, manager of clinical research cluster), senior medical advisor (SS, pediatric neuro-metabolic specialist) with experience in hospital leadership and an evaluator (WG) to measure family and 


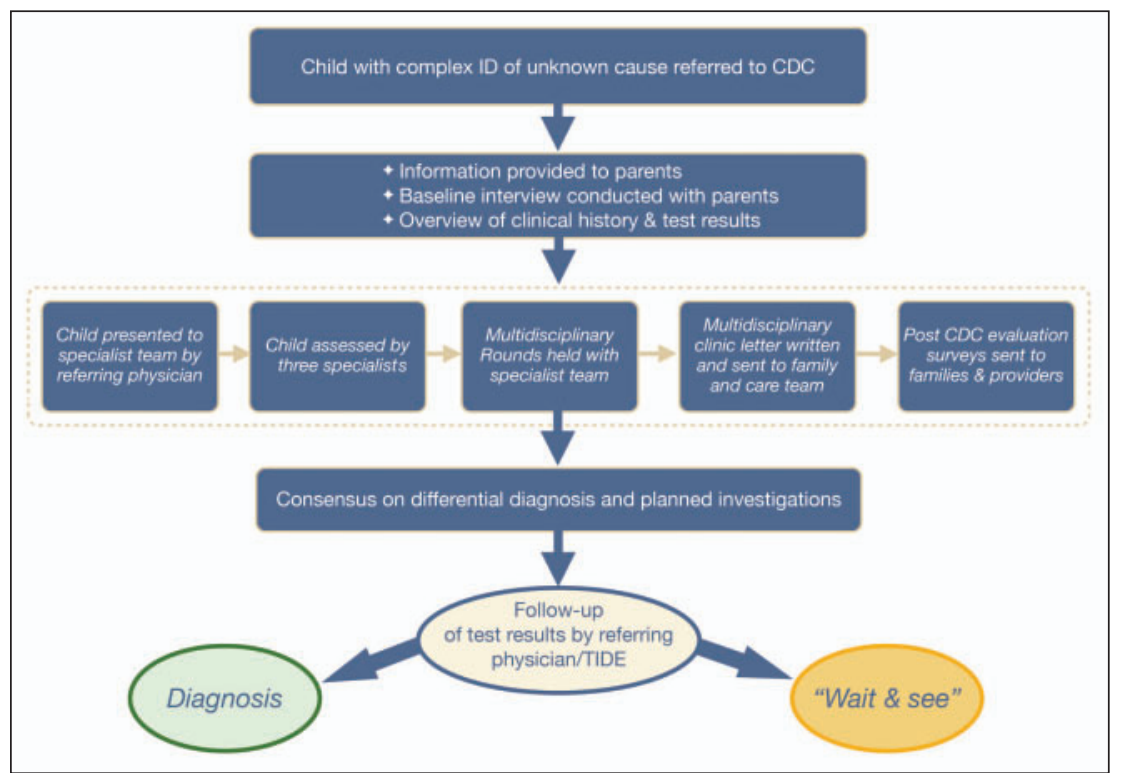

Figure 1: Structure and organization of the TIDE Complex Clinic. Before the clinic, the manager counsels the family about goals and expectations and obtains informed consent; the evaluator interviews the family. Thirty minutes prior to the clinic, the referring physicians present their patient to the evaluating specialists, who subsequently circulate, assessing each patient for 45-60 minutes. The manager then meets with each family to followup, explain next steps and answer any additional questions. The CDC team, referring physicians, evaluating specialists, and other invited multidisciplinary medical professionals and researchers attend one-hour rounds (continuing medical education accredited) immediately following the clinic. The rounds moderator briefly summarizes the clinical presentation and test results of each case and asks for input from each evaluating specialist on the most salient findings and possible diagnoses. With the input of other professionals at the rounds, a consensus plan is formulated, which includes a differential diagnosis, further investigations and follow-up.

provider satisfaction. This team is supported by an interdisciplinary clinician/specialist collaborative team, with administrative assistance provided by clerks/research coordinators.

The CDC Clinical Team includes lead physicians from the divisions of Biochemical Diseases, Pediatric Neurology, Psychiatry, Developmental Pediatrics and Medical Genetics who assess patients in the CDC on a regular basis. Furthermore, diverse subspecialist physicians participate in the CDC on an "as needed" basis according to the patient's phenotype and problems, including Sleep Medicine, Rheumatology, Hematology, Neuroradiology and Gastroenterology. Additionally, a biochemical geneticist, a cytogeneticist, a molecular geneticist, and a bioinformatician contribute to case discussions at CDC postclinic rounds.

The CDC organization and patient flow are illustrated in Figure 1. The CDC takes place once every two months. The CDC director receives referrals from three core divisions at $\mathrm{BCCH}$. Patient inclusion criteria are defined as: 1) ID confirmed via formal and/or clinical neurocognitive assessments plus complex medical symptoms of unknown etiology despite extensive diagnostic work up (defined as a minimum of: clinical evaluation by two specialists, chromosome micro-array; TIDE 1st tier testing; additional metabolic testing and/or neuroimaging); 2) strong suspicion of a genetic etiology based on clinical phenotype, test results, family history; 3) neurologic and/or systemic co-morbidity (including dysmorphic features); 4) interest of patient/family to attend the CDC. The director selects patients for the clinic and arranges for physicians from three specialties (most relevant for the specific phenotype) to assess the patient, including second opinions. To optimize efficiency, when possible, three patients requiring input from the same set of specialists are scheduled for each CDC.

The director and manager coordinate the preparation of a summary of diagnostic testing/relevant clinical information, together with several patient specific questions to each consulting service. These summaries are circulated to consulting specialists one to two weeks prior to the CDC.

The schedule of the CDC is outlined in the legend of Figure 1. With parental consent, photos and videos of patients are collected and accessible via a secure intranet drive to facilitate follow-up and further discussions.

One week after the CDC, the director prepares a summary of the group's consensus for each patient. The information is sent in the form of a one-page letter to the referring physician, all attending specialists, paediatrician, family physician, and family. Each specialist also prepares a consulting letter that is distributed among the same group. Clinical information is entered into the REDCap (Research Electronic Data Capture) secure database. The referring physician is responsible for follow-up on planned tests and investigations, and updates the director on the diagnostic status of each patient. This results in either a diagnosis with appropriate referrals, or a 'wait and see' approach, i.e. patient re-evaluation two to three years later, when more relevant genetic and clinical information may be available.

Data analyses: Supplementary Methods (at end of this article) provides information on: 1) costs assessment and comparison of the diagnostic work-up prior to and during the clinic, both to the health care system and to the families; 2) tools used for qualitative evaluation of the CDC experience for medical specialists and families; 3) continuous quality improvement.

Ethics: The institutional review board at $\mathrm{BCCH}$ and the University of British Columbia, Vancouver, Canada approved the study protocols [H11-01142; H11-01530, H12-00067]. All parents/ legal guardians were asked for informed consent for participation in the larger TIDE study, CDC evaluation study and publication of these results. 
Table 1: Phenotype and diagnostic status of patients evaluated at the Complex Diagnostic Clinic (CDC)

\begin{tabular}{|c|c|c|c|c|c|c|c|}
\hline Pt. & Sex & Age & $\begin{array}{l}\text { Severity } \\
\text { of ID }\end{array}$ & Type & Confirmed Diagnosis & Working Diagnosis & Relevant Diagnostic Test \\
\hline 1 & $\mathrm{f}$ & $5 y$ & moderate & ID + epilepsy & POLG1 encephalomyopathy* & & POLG1 \\
\hline $2 \S$ & $f$ & $15 \mathrm{y}$ & moderate & ID+ epilepsy & $\begin{array}{l}\text { de novo deletion } 19 \mathrm{p} 13.2,595.5 \mathrm{~Kb} \\
(D N M 2, D N M T 1)\end{array}$ & $\begin{array}{l}\text { variant Bardet-Biedl } \\
\text { syndrome }(B S S 1)\end{array}$ & $\begin{array}{l}\text { CMA + whole exome } \\
\text { sequencing }\end{array}$ \\
\hline $3 \S$ & $\mathrm{m}$ & $13 y$ & mild & ID+ epilepsy & none & $\begin{array}{l}\text { variant Bardet-Biedl } \\
\text { syndrome }(B S S 1)\end{array}$ & whole exome sequencing \\
\hline 4 & $\mathrm{f}$ & $10 \mathrm{y}$ & borderline & ID + behavioral difficulties & $\begin{array}{l}\text { familial basal ganglia calcifications* } \\
\& \text { maternally inherited duplication } \\
\mathrm{Xp} 22.31,1.63 \mathrm{Mb} \text { (3 copies) }\end{array}$ & none & CMA \\
\hline 5 & $\mathrm{~m}$ & $\begin{array}{l}17 \\
\text { mo. }\end{array}$ & profound & DD+ dysmorphic features & $\begin{array}{l}\text { X-linked alpha-thalassemia } \\
\text { syndrome * (p.R246C mutation in } \\
\text { exon } 9 \text { of } A T R X)\end{array}$ & none & $A T R X$ \\
\hline 6 & $\mathrm{~m}$ & 24 mo. & profound & DD+ failure to thrive & $\begin{array}{l}\text { Glycogen Storage Disease IXa } \\
*(2213 \mathrm{del} C \text { in exon } 20 \text { of } P H K A 2)\end{array}$ & none & $P H K A 2$ \\
\hline 7 & $\mathrm{~m}$ & $10 y$ & mild & ID+ autism & none & none & none \\
\hline 8 & $\mathrm{~m}$ & $13 y$ & moderate & ID + dysmorphic features & 15q26.1 Microdeletion Syndrome & none & CMA \\
\hline 9 & $\mathrm{f}$ & $4 y$ & profound & DD+congenital anomalies & Methotrexate embryopathy* & DSEL deficiency & $\begin{array}{l}\text { clinical exam \& whole } \\
\text { exome sequencing }\end{array}$ \\
\hline 10 & $\mathrm{~m}$ & $7 y$ & moderate & ID + multisystem disease & none & $M F N G$ deficiency & whole exome sequencing \\
\hline 11 & $\mathrm{~m}$ & $6 y$ & profound & $\begin{array}{l}\text { ID+ congenital brain } \\
\text { anomalies }\end{array}$ & none & $D S C A M L 1$ deficiency & whole exome sequencing \\
\hline 12 & $\mathrm{f}$ & $9 \mathrm{mo}$. & moderate & $\mathrm{DD}+$ growth retardation & none & Crisponi Syndrome & whole exome sequencing \\
\hline 13 & $\mathrm{~m}$ & $3 y$ & moderate & DD+ neurologic deficits & none & $\begin{array}{l}\text { mitochondrial disease* } \\
\text { (mtDNA mutation) }\end{array}$ & mtDNA sequencing \\
\hline 14 & $\mathrm{f}$ & $16 y$ & moderate & $\begin{array}{l}\text { ID+ psychiatric } \\
\text { disturbances }\end{array}$ & none & none & none \\
\hline 15 & $\mathrm{~m}$ & $16 y$ & moderate & ID+ dysmorphic features & none & Costello syndrome* & $\begin{array}{l}\text { clinical exam (gene / panel } \\
\text { funding refuted) }\end{array}$ \\
\hline 16 & $\mathrm{f}$ & $7 y$ & mild & ID + neurologic deficit & none & Myotonic Dystrophy & ZNF9 (gene test refuted) \\
\hline 17 & $\mathrm{f}$ & $10 \mathrm{y}$ & moderate & $\begin{array}{l}\text { ID+ psychiatric } \\
\text { disturbances }\end{array}$ & none & $\begin{array}{l}\text { overgrowth syndrome } \\
(P T E N-\text { related })^{*}\end{array}$ & gene panel funding refuted \\
\hline 18 & $\mathrm{f}$ & $18 \mathrm{y}$ & mild & ID+ epilepsy & none & none & none \\
\hline 19 & $\mathrm{f}$ & $15 y$ & moderate & ID+ epilepsy & none & none & none \\
\hline 20 & f & $14 \mathrm{mo}$ & moderate & DD+ neurologic deficit & Smith Magenis syndrome* & none & $R A I 1$ \\
\hline 21 & $\mathrm{f}$ & 18 mo. & profound & DD+ epilepsy & Congenital Rett Syndrome* & none & $F O X G 1$ \\
\hline 22 & f & $3 y$ & mild & DD+ dysmorphic features & Sotos syndrome* & none & clinical exam \\
\hline 23 & $\mathrm{f}$ & $3.5 y$ & moderate & DD+ dysmorphic features & none & Triple AAA syndrome* & $\begin{array}{l}\text { clinical exam \& endocrine } \\
\text { test }\end{array}$ \\
\hline 24 & $\mathrm{f}$ & $4 y$ & moderate & DD+ microcephaly & Angelman syndrome* & none & clinical exam \& UBE3A \\
\hline
\end{tabular}

§Patients \# 2 and \#3 are the sibling pair. * Diagnosis established based on clinical expertise directing specific test; for all other diagnoses, clinical expertise was required to interpret CMA and/or WES. Abbreviations: Pt.=patient; $\mathrm{CMA}=$ chromosome microarray analysis; $\mathrm{y}=\mathrm{years}$; mo.=months; mtDNA=mitochondrial DNA

\section{RESULTS}

\section{Patients}

In total, 27 patients were referred to the CDC during a 16month period, of which three did not meet the criteria (sibling pair with unspecific ID, i.e. lacking other neurologic / systemic symptoms; one patient with insufficient previous work-up). Table 1 provides an overview of the 24 patients (one sibling pair: \#2 and \#3) consecutively evaluated in seven Clinics over 16 months: 12 patients were referred by Biochemical Diseases, nine by Neurology, and three by Medical Genetics. The phenotypic presentations were classified according to the most dominant comorbidity; patients were most often referred for severe epilepsy, neurologic impairments (e.g. movement disorder) and dysmorphic features. The male to female ratio was 9 to 15 ; this is notable, given the greater incidence of ID in males. No reasons other than chance alone were identified for this female predominance. The average age of these patients was 7.9 years (range 9 months-18 years). The average age of each patient's first consultation at $\mathrm{BCCH}$ was 1 year 11 months: the average interval between the patient's first consultation at $\mathrm{BCCH}$ and the $\mathrm{CDC}$ was roughly six years. The mean number of specialists 
Table 2: Overview of all diagnostic tests performed prior to and during the Complex Diagnostic Clinic

\begin{tabular}{|c|c|c|c|c|c|c|c|c|}
\hline Pt. & \#spec & CMA & $\begin{array}{l}\text { TIDE } \\
1^{\text {st }} \text { tier } \\
\text { tests* }\end{array}$ & $\begin{array}{l}\text { Addition } \\
\text { metabolic } \\
\text { tests }\end{array}$ & Cytogenetic & Specific Molecular Tests & WES & $\begin{array}{l}\text { Neuro- } \\
\text { Imaging }\end{array}$ \\
\hline 1 & 4 & yes & $\mathrm{N}$ & 8 & -karyotype & $\begin{array}{l}\text {-DMPK, FMR1, POLG1 (plus dup/del), SLC2A1 (plus MLPA), UBE3A } \\
\text { - mtDNA genome with del. \& depl. analysis } \\
\text {-15q11-13 methylation analysis }\end{array}$ & no & $\begin{array}{l}2 \mathrm{MR} \\
2 \mathrm{CT}\end{array}$ \\
\hline 2 & 4 & yes & $\mathrm{N}$ & 11 & $\begin{array}{l}\text {-karyotype } \\
\text { - FISH; 2q11, } \\
\text { long arm chr.2 }\end{array}$ & $\begin{array}{l}\text {-MELAS, MERRF, NARP (mtDNA targeted mutation analysis) } \\
\text {-HFE (C282Y \& H63D variants) }\end{array}$ & yes & $\begin{array}{l}5 \mathrm{MR} \\
3 \mathrm{CT}\end{array}$ \\
\hline 3 & 7 & yes & $\mathrm{N}$ & 0 & -karyotype & -none & yes & $3 \mathrm{MR}, \mathrm{CT}$ \\
\hline 4 & 5 & yes & $\mathrm{N}$ & 11 & -none & $\begin{array}{l}\text {-MELAS, MERRF, LOHN, NARP (mtDNA targeted mutation } \\
\text { analysis) }\end{array}$ & no & $\mathrm{MR}, \mathrm{CT}$ \\
\hline 5 & 4 & yes & $\mathrm{N}$ & 2 & -none & -ATRX & no & $\mathrm{MR}, \mathrm{CT}$ \\
\hline 6 & 2 & yes & $\mathrm{N}$ & 2 & -karyotype & $-P H K A 2$ & no & none \\
\hline 7 & 4 & yes & $\mathrm{N}$ & 4 & -karyotype & $-F M R 1, S L C 2 A 1$ (plus MLPA) & no & MR/MRS \\
\hline 8 & 6 & yes & $\mathrm{N}$ & 5 & $\begin{array}{l}\text {-karyotype } \\
\text {-FISH; } 22 \mathrm{q} 11 \\
22 \mathrm{q} 13\end{array}$ & $\begin{array}{l}\text {-MELAS (targeted mutation analysis) } \\
\text {-FMR1 } \\
\text {-whote mtDNA genome with del. analysis }\end{array}$ & no & MRS \\
\hline 9 & 9 & yes & $\mathrm{N}$ & 4 & -karyotype & $\begin{array}{l}-C P T 1 A, \mathrm{c} .1436 \mathrm{C}>\mathrm{T}(\mathrm{p} 479 \mathrm{~L}) \\
-C O L 1 A 1, C O L 1 A 2\end{array}$ & yes & $\begin{array}{l}\text { MRS } \\
3 \mathrm{CT}\end{array}$ \\
\hline 10 & 8 & yes & $\mathrm{N}$ & 6 & -none & $\begin{array}{l}\text {-FMR1, FOXP3 } \\
\text {-MERF, MELAS (targeted mutation analysis) } \\
\text { - whole mtDNA genome with del. analysis }\end{array}$ & yes & MR/MRS \\
\hline 11 & 10 & yes & $\mathrm{N}$ & 10 & $\begin{array}{l}\text {-karyotype } \\
\text {-FISH; 5q11-13, } \\
22 \mathrm{q} 11\end{array}$ & $\begin{array}{l}-A R X, C D K L 5, M E C P 2, P H G D H \\
\text {-15q11-13 methylation analysis }\end{array}$ & yes & $\begin{array}{l}2 \mathrm{MRS} \\
2 \mathrm{MR} \\
3 \mathrm{CT}\end{array}$ \\
\hline 12 & 8 & yes & $\mathrm{N}$ & 3 & -karyotype & -none & yes & $\mathrm{MR} / \mathrm{MRS}$ \\
\hline 13 & 5 & yes & $\mathrm{N}$ & 10 & -karyotype & $\begin{array}{l}\text { - } C 10 O R F 2, P H D G H \\
\text {-repeat analysis of } A T X N 1, A T X N 2, A T X N 3, C A C N A 1 A \& A T X N 7 \\
\text {-whole mtDNA genome }\end{array}$ & no & MR/MRS \\
\hline 14 & 4 & yes & $\mathrm{N}$ & 7 & $\begin{array}{l}\text {-karyotype } \\
\text {-FISH; 15q11-13 }\end{array}$ & $\begin{array}{l}-F M R 1, N P C 1, N P C 2, O T C, S L C 2 A 1 \text { (plus MLPA) } \\
-15 q 11-13 \text { methylation analysis }\end{array}$ & no & $\begin{array}{l}3 \mathrm{MR} \\
\mathrm{CT}\end{array}$ \\
\hline 15 & 8 & yes & $\mathrm{N}$ & 3 & $\begin{array}{l}\text {-karyotype } \\
\text {-FISH; } 7 \mathrm{q} 11.23\end{array}$ & $-F M R 1, H R A S$ & no & $\begin{array}{l}\text { MRS } \\
2 \mathrm{CT}\end{array}$ \\
\hline 16 & 3 & yes & $\mathrm{N}$ & 0 & -FISH; 22q11 & $-D M P K$ & no & MR \\
\hline 17 & 6 & yes & $\mathrm{N}$ & 7 & $\begin{array}{l}\text {-karyotype } \\
\text {-FISH; 5q35, } \\
\text { 5q15.2, } \\
\text { Subtelomeres }\end{array}$ & -EZH2, FMR1, NK5, NPC1, NPC2 & no & MR/MRS, CT \\
\hline 18 & 5 & yes & $\mathrm{N}$ & 4 & -karyotype & $-E P M 1$ (repeat analysis) & no & $\begin{array}{l}\mathrm{MR} / \mathrm{MRS} \\
2 \mathrm{CT}\end{array}$ \\
\hline 19 & 5 & yes & $\mathrm{N}$ & 2 & -karyotype & -none & no & $2 \mathrm{MR}$ \\
\hline 20 & 2 & yes & $\mathrm{N}$ & 2 & -none & -none & no & none \\
\hline 21 & 8 & yes & $\mathrm{N}$ & 9 & $\begin{array}{l}\text {-karyotype, } \\
\text {-FISH; } 15 \mathrm{q} 11-13\end{array}$ & $\begin{array}{l}-S L C 2 A 1 \text { (plus MLPA) } \\
-15 \mathrm{q} 11-13 \text { methylation analysis }\end{array}$ & no & MR \\
\hline 22 & 4 & yes & $\mathrm{N}$ & 4 & $\begin{array}{l}\text {-karyotype } \\
\text {-FISH; 15q11-13 }\end{array}$ & $\begin{array}{l}-15 \mathrm{q} 11-13 \text { methylation analysis } \\
-N S D 1 \text { funding denied }\end{array}$ & no & MR \\
\hline 23 & 4 & yes & $\mathrm{N}$ & 1 & $\begin{array}{l}\text {-karyotype } \\
\text {-FISH 15q11-13 }\end{array}$ & $\begin{array}{l}-C H D 7 \\
-15 q 11-13 \text { methylation analysis }\end{array}$ & yes & MRS \\
\hline 24 & 3 & yes & $\mathrm{N}$ & 0 & $\begin{array}{l}\text {-karyotype } \\
\text {-FISH 15q11-13 }\end{array}$ & $\begin{array}{l}-U B E 3 A \\
-15 q 11-13 \text { methylation analysis }\end{array}$ & no & MR \\
\hline
\end{tabular}

*TIDE 1st tier test: blood plasma amino acids, total homocysteine, bloodspot acylcarnitine profile ammonia, lactate \& urine oligosaccharides, mucopolysaccharides, purines/pyrimidines, cretaine matabolites (online Figure S1). Abbreviations: CMA=chromosome micro-array analysis; CT=Computed Tomography imaging of brain; FISH= Fluorescence In Situ Hybridization; MLPA=Multiplex Ligation-dependent Probe Amplification; mtDNA=mitochondrial DNA; MR=magnetic resonance imaging of the brain+/- $\operatorname{spectroscopy}(\mathrm{S})$; \#spec=number of specialists who evaluated patient prior to CDC; WES=Whole Exome sequencing; 
who evaluated the patient throughout this time period was five (range 2-10).

\section{Diagnostic Tests}

Table 2 provides an overview of all investigations performed on the 24 patients seen prior to and during the CDC. Chromosome microarray analysis was performed for all patients; this is appropriate as this test has the highest diagnostic yield in ID (up to 20\%). All patients except two underwent neuroimaging. Sequential single gene tests and mitochondrial DNA sequencing were the next most commonly ordered tests. First tier TIDE Protocol (supplementary Figure S1) testing was completed in all patients, including those who currently remain without a diagnosis. Additionally, various biochemical tests were done (up to 11 per patient). Whole exome sequencing (WES) was initiated in seven cases, one of which received government-approved funding due to the severity of her condition and long-term hospital admission. The remaining six were funded through the TIDEX gene discovery study. Finally, funding for molecular tests was refuted by the government health insurance in four patients, impeding confirmation of diagnosis in all.

\section{Diagnostic Yield}

In the 24 patients evaluated during a 16-month period in the CDC, causal diagnoses were firmly established in $11(46 \%)$. In another nine $(38 \%)$ patients, strong differential or working diagnoses were formulated; gene defects via WES (with mutations confirmed to segregate with disease in the family) are in the process of biological validation $(n=6)$ while funding for gene tests to confirm known diagnosis were refuted in others $(n=4)$. Three patients currently remain without a diagnosis; each would be a candidate for WES if this test were funded in our system (Tables $1 \& 2$ ).

At the time of writing, two separate diagnoses are suspected in three patients: causal: Case \#2 was diagnosed with a CNV, with DNM2 and DNMT1 deletions explaining her epilepsy. However her other features, shared with her brother, include severe psychiatric disturbances, short stature, hypogonadism and dysmorphisms, are likely due to variant Bardet-Biedl syndrome; WES-identified missense mutations in the BSSl gene are currently being validated. For patient \#4, the CDC consensus comprised benign familial basal ganglia calcifications as 'diagnosis per exclusionem', and maternal inherited Xp22.31 duplication predisposing her to a learning disorder. For patient \#9, who has multiple congenital anomalies, Methotrexate Embryopathy was considered an incomplete explanation for her recurrent fractures. Upon re-examination during the CDC, bluish sclerae and translucent skin were found and are suggestive of osteogenesis imperfecta, prompting further studies yielding abnormal (pro-)collagen results of skin biopsy analysis and a candidate gene via WES (see Supplementary Results). Finally, for the latter patient as well as for cases \#20, \#22 and \#24, the diagnosis was already suspected prior to the clinic; thus in the remaining 16 patients $(67 \%)$ the clinic's added value was most significant in terms of identifying a new diagnosis.

A treatable inborn errors of metabolism (IEM) was diagnosed in one patient (\#6): glycogen storage diseases IXa is not one of the 81 treatable IDs generated by our systematic review, as ID is present in less than half of patients. However, for this patient we suspect that subclinical recurrent metabolic disturbances with hypoglycaemia, ketosis and elevated liver transaminases caused energy failure and liver damage. Since he has been on dietary therapy, his somatic growth, fine and gross motor skills have normalized for his age, however his speech and communication remain mildly delayed.

CMA contributed to a confirmed diagnosis in three cases; for the female sibling described previously, and for two patients (\#4, \#8), CNVs hitherto dismissed as insignificant were reanalyzed during the CDC and deemed etiologic. Further, single gene tests prompted by phenotypic findings, yielded three confirmed diagnoses, reflecting the diagnostic acuity of our group. Whole exome sequencing identified the causal gene in five patients, one of which is confirmed and the remaining four in various stages of validation. In total, two gene defects were described for the first time in humans (\#13,\#14). In conclusion, the clinical exam with detailed phenotyping proved essential to establish a strong working diagnosis $(\mathrm{n}=5)$, and subsequently directed the appropriate test for confirmation thereof $(n=9)$; and/or (re-) interpreted the results of WES and CMA $(n=7)$.

\section{Added value through multi-specialist evaluation}

Aside from the high diagnostic yield, the true value of the collaborative approach for establishing a diagnosis through integrated clinical and laboratory expertise of a group of specialists is best illustrated by the eight case vignettes described in Supplementary Results.

\section{Costs of CDC versus previous practice}

Detailed cost analyses were performed for 23 patients (one was excluded due to extensive inpatient stays and complex symptomatology that did not allow for classification of diagnostic evaluation versus care management). Figure 2 compares the average cost per patient prior to the clinic, versus during the clinic (including all tests ordered). While clinical consultation represents about a third of the total cost in both scenarios, the CDC provides consultation at a quarter of the cost compared with the traditional model of diagnostic care. Travel costs and lost income for families are reduced by $96 \%$ in the TIDE-BC CDC model of diagnostic care.

The CDC's most significant expense is clinic coordination (including selection of referrals, inviting specialists, preparation of information to be sent to evaluating specialists, drafting clinic letters and following up on results), representing nearly a third of the total costs.

\section{Patient Satisfaction}

Pre-clinic interviews were conducted with half $(n=12)$ of the families evaluated in the TIDE-BC CDC. All families emphasized the importance of a more coordinated approach to finding a diagnosis for their child. One-third expressed their exhaustion with trying to find a diagnosis and the stress associated with taking on the role of a 'care coordinator', and simultaneously the hope for improvement via a collaborative multi-specialist evaluation. None expected a diagnosis or change in their family situation as a result of the clinic. A third of families spoke of their hopes for additional school supports and 


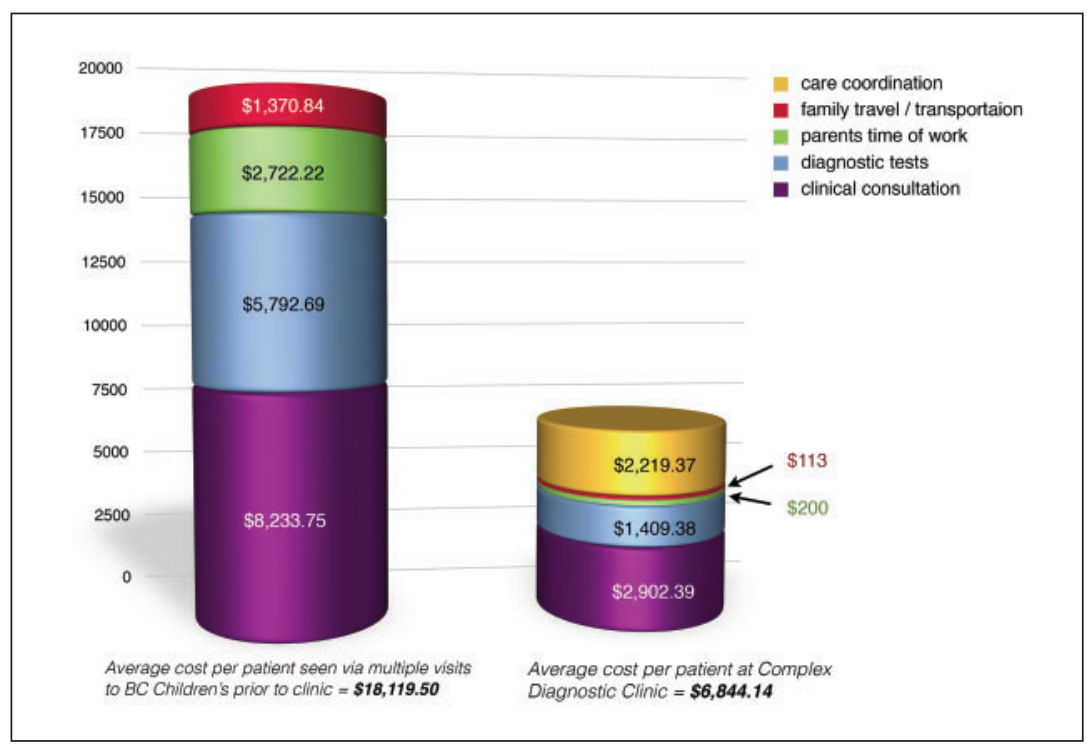

Figure 2: Average cost per patient of diagnostic evaluation prior to the Complex Diagnostic Clinic $(C D C)$, versus during the $C D C$. The pre-clinic total includes the cost of family travel/ transportation, parents time off work, diagnostic tests (laboratory and other diagnostic procedures), and clinical consultation. The Complex Diagnostic Clinic total includes the cost of coordinating the clinic, one trip to hospital, one day off work for a parent, costs of diagnostic tests ordered from clinic, and the cost of clinical consultation (including follow-up time by specialists to order testing).

two expressed a desire to understand the transition to youth/adult care for their child and were hopeful the clinic could provide some answers to guide the process.

Post clinic evaluation: 14 families out of a possible 22 (one sibling pair and one family who chose not to be sent the postclinic survey) took part in the online post-clinic survey.

All families reported a high degree of satisfaction with the overall experience (scoring excellent, very good or good), and would recommend the TIDE-BC CDC. The significance of an established diagnosis for an individual family may be illustrated by this mother's quote: "Understanding the gene that is responsible for my son's condition because of coming to the Clinic is a huge relief. I can finally stop blaming myself for something I may have done while I was pregnant. After six years of guilt, it feels awesome to know what's going on. We still have a huge journey ahead but now I can let go."

Families valued the CDC as one-stop clinic (mean rating score 9/10) for their child to receive state of the art diagnostic assessment. Interestingly, families provided a somewhat lower score for confidence that a treatable cause $(7 / 10)$ had been ruled out.

The majority found the CDC to be an improvement over their previous experiences, particularly with respect to their confidence and trust in care (half report an improvement), consistency of information (two-thirds) and ease of obtaining access to specialists (nearly three quarters).

\section{Provider Satisfaction}

Specialists $(n=21)$ were satisfied with the CDC, confirming it had successfully achieved its goals, and felt the clinic to be an effective mechanism for supporting multidisciplinary provision of care, enabling a faster time to diagnosis (mean rating score 9/10). "Coming together face-to-face to discuss complex cases is so valuable for patient care. We are collectively making diagnoses that we would likely not otherwise have been able to do working alone, or would have taken years." Finally, the CDC increased the physicians' knowledge about (novel) genetic causes of ID as well as the application of WES as diagnostic test (score 9/10), and was rated as an excellent vehicle for resident and fellow learning opportunities. Attendance at the rounds has been consistently high, with an average participation of 15 clinical / laboratory specialists at each.

\section{Discussion}

To our knowledge this is the first detailed report of yield, costs and patient / provider satisfaction of a multi-disciplinary clinic dedicated to the diagnosis of rare etiologies in ID patients with complex co-morbid features, since the advent of genomic and high-throughput technologies.

However, the following limitations must be acknowledged: 1) the small size of the study group seen during the pilot phase of the CDC during pilot phase and reported here (with no clear explanation for the surprising female predominance in this group of consecutive CDC patients); 2) the retrospective nature of the chart reviews with inherent incompleteness, however, the fact that these were performed by one experienced research assistant under auspices of the medical director increased its reliability; 3 ) multi-purpose use of some investigations (for diagnosis versus management or both), e.g. EEG used to identify seizure type and to monitor treatment effects, in which the medical director made the decision regarding categorization; 4) the essential contribution of some tests performed prior to the CDC in establishing a diagnosis during the CDC, together with the exclusion of costs of logistics of clinic visits prior to the CDC (preparations by the nurse, administrative assistant, specialist), which both preclude the analysis of avoidable pre-CDC 'costs and/or diagnostic delay'; 5) test results still pending for four patients, which could confirm additional diagnoses, thereby 
increasing the effectiveness of the CDC beyond that reported here; 6) experimental data and identification of phenotypically similar patients with different mutant alleles are required for validation of novel gene defects identified in patients \#9-11 (studies still in progress); 7) funding applied for but refuted by the provincial health insurance because the "outcome of testing does not lead to therapeutic interventions, and as a result lack of diagnostic confirmation in four patients; 8) the cost analysis being limited to the specific qualities of the healthcare system and setting of a large Canadian province.

Despite these limitations, the CDC clearly illustrates the strength of uniting specialists in person for collaborative decision-making, as evidenced by our diagnostic success rate as well as the level of patient/family and provider satisfaction. The high yield (46\% with confirmed diagnosis, and another 38\% with a strong working diagnosis) achieved for this group of extensively tested ID patients with complex symptomatology of unknown cause, exceeded our expectations given reported yields of $40-50 \%$ in ID patients with little or no pre-referral testing, as outlined below. For each diagnosis, working or confirmed, clinical phenotyping and multi-disciplinary expertise were essential to identify a syndrome, direct the single gene test and/or (re-) interpret complex CMA and WES data with the inherent multitude of variants of unknown significance.

For our highly challenging patient group, no (recent) data in the literature exist to provide a comparison. Engbers et al reported a causal diagnosis in $12.9 \%$ of 433 ID patients evaluated in a multi-specialist tertiary clinic in the Netherlands ${ }^{17}$. These results are not completely comparable to ours, however, given the complexity of the phenotypes and number of prereferral tests were fewer, and CMA and WES were unavailable The study did demonstrate the strength of rigorous, multispecialist evaluation; although metabolic tests had been performed in 376 (87\%) of 433 children, additional IEMs were still diagnosed in $2.8 \%$. Comparison with other studies reporting the diagnostic yield in large groups of ID patients, e.g. $50 \%$ by van Karnebeek et al in $2005^{7}, 40 \%$ by Rauch et al in $2006^{18}$, and more recently in 2011-12 from China and India ${ }^{19,20}$, is challenging for similar reasons, plus the fact that these were performed in a single rather than a multi-specialist setting.

In addition to its diagnostic success, the CDC also demonstrates that one clinic encompassing multidisciplinary collaboration can accomplish a process that would normally take months and involve numerous consultations. For all patients previously available CMA results were re-interpreted by multiple specialists using updated database information; variants of unknown significance were deemed pathogenic in three. In general, the CDC provides a more streamlined evaluation for children with complex needs. Most importantly, 11 children and families now benefit from the advantages of a confirmed diagnosis, including improved genetic counselling, access to community resources, management, and prognosis. The most profound impact of a CDC diagnosis (previously eluding both metabolic and gastroenterology specialists) was observed for the glycogen storage disease IXa patient, with improved psychomotor development after initiation of appropriate treatment. Additionally, another nine patients with a strong working diagnosis experienced similar benefits, albeit with less certainty at this time (six of whom face the possibility of confirmation via pending test results).
Despite various limitations of the cost analyses, it is clear that due to the high diagnostic yield, the CDC can provide a diagnosis at more value per dollar than traditional methods. Figure 2 illustrates the substantial investment incurred in pursuing a diagnosis for each patient prior to the clinic, but did not generate a diagnosis (traditional model). Not only does the CDC reduce the impact on the healthcare system, (i.e. reduced tests, reduced time to diagnosis and fewer consultations) but it also eliminates multiple trips to the hospital, reducing lost worktime for parents.

Figure 2 also shows the costs of organizing the CDC. In the future, as the workflow is streamlined, and the medical director (pediatric subspecialist) transfers more responsibilities to the clinic manager, these costs should decrease. However, inherent to its aim of solving a complex mystery, the CDC will remain a work- and time-intensive endeavour. If funding allows, a genetic counsellor would be a valuable addition to our team, to organize sample collection and investigations (including follow-up of results), aid the explanation of complex tests and in the disclosing of results, as well as provide ongoing counseling of families either receiving a molecular confirmation, or continuing to deal with the uncertainty of an unknown diagnosis. Our individualized approach, with positive evaluations from both patients and providers, highlights that for a particular subset of ID patients, the CDC is an exemplary model of personalized diagnosis and platform for translational medicine. Ideally in the future, complex ID patients will be seen in the CDC during an earlier stage of their diagnostic evaluation to save costs via earlier diagnosis and more targeted testing.

Further, the TIDE-BC CDC has created a multi-specialist panel for interpretation of WES data to efficiently define candidate genes and plan for validation studies. Once this test becomes clinically funded, the $\mathrm{CDC}$ is poised to elevate the profile of $\mathrm{BCCH}$ and to optimize the diagnostic yield and (cost) effectiveness to benefit patients, families, the health care system and society as a whole. This framework can readily be implemented in other areas in pediatrics, such as rheumatology and immunology.

\section{CONClusions}

In the current age of high throughput or -omics technologies, the close interaction of physicians and laboratory scientists, clinicians and researchers, and care providers and patients is pivotal for interpretation and translation of complex genomic and biochemical data into meaningful information to improve the health of children affected by rare diseases ${ }^{21}$. The CDC has created an environment to allow such synergy and to provide the framework for future medicine. This strength will help the CDC move from the current research realm to the hospital's core clinical service, perhaps serving other complex patient populations as well, and continuously adapting to new technologies, knowledge and care demands. Finally, in the realm of rare diseases, the importance of international collaborations for gene discoveries and delineation of phenotypes and disease mechanisms is clearly illustrated by the Canadian FORGE and U.S. Mendelian Centers for Genomics programs ${ }^{22,23}$; the TIDE CDC continues to benefit as well as contribute to this exciting process. 


\section{ACKNOWLEDGMENTS}

TIDE CDC Team: Linlea Armstrong ${ }^{3,7}$, Susan Baer $^{3,8}$, Maria Boldut $^{1-3}$, Vann Chau ${ }^{2,3,5}$, Jean-Paul Collet ${ }^{2,3}$, Michelle K. Demos $^{2,3,5}$, Patrice Eydoux ${ }^{3,9}$, Robin Friedlander ${ }^{3,8}$, Gabriella A. Horvath $^{1-3}$, Osman S. Ipsiroglu ${ }^{2,3,10}$, Mir Lafek ${ }^{1-3}$, Anna M. Lehman $^{3,4,7}$, M.E. Suzanne Lewis ${ }^{3,4,7}$, Barbara McGillivray ${ }^{3,7}$, Tanya N. Nelson ${ }^{3,9}$, Millan S. Patel ${ }^{3,4,7}$, Ramona Salvarinova ${ }^{1-3}$, Kathryn Selby ${ }^{2-5}$, Christèle du Souich ${ }^{3,7}$, Hilary D. Vallance ${ }^{3,9}$, Margot I. van Allen ${ }^{3,7}$, Wyeth W. Wasserman $3,4,7,11$

Affiliations: ${ }^{1}$ Division of Biochemical Diseases, ${ }^{2}$ Department of Pediatrics; ${ }^{3}$ Treatable Intellectual Disability Endeavour in British Columbia (www.tidebc.org); ${ }^{4}$ Child and Family Research Institute; ${ }^{5}$ Division of Neurology; ${ }^{6}$ Howegroup Public Sector Consultants Inc.; ${ }^{7}$ Department of Medical Genetics; ${ }^{8}$ Division of Child and Adolescent Psychiatry; Department of Psychiatry; ${ }^{9}$ Department of Pathology \& Laboratory Medicine; ${ }^{10}$ Division of Developmental Pediatrics; ${ }^{11}$ Center for Molecular Medicine and Therapeutics; B.C. Children's Hospital, University of British Columbia, Vancouver, B.C., Canada

The authors thank the patients and their families for participating in the CDC and TIDE studies; the specialists and nurses contributing their expert input; all those involved for their valuable feedback; Dr. J. Friedman (clinical geneticist) for his suggestions regarding the organization of the CDC; Dr. G. Sinclair (biochemical geneticist) for interpretation and reporting of biochemical laboratory results; Dr. E. Mickelson (developmental pediatrician) for providing results of developmental / cognitive assessments; Dr. W. McKellin (medical anthropologist) for his input on qualitative evaluation of the CDC experience; Mrs. C. Sowerbutt (medical writer) for manuscript edits; and Mrs. R. Giesbrecht, Mrs. M. Sebastiano, and Mrs. M. Higginson for their contributions to the CDC organisation and administration.

\section{FUNDING}

This work was conducted as part of the TIDE-BC project (Treatable Intellectual Disability Endeavour in British Columbia- www.tidebc.org), funded by the B.C. Children's Hospital Foundation (Vancouver, Canada) as the 1st Collaborative Area of Innovation.

\section{REFERENCES}

1. Shalock RL, Borthwith-Duffy S, Buntinx WHE, Coulter DL, Craig EM. Intellectual disability: definition, classification and systems of supports. 11th ed. Washington D.C.: American Association on Intellectual and Developmental Disabilities; 2011.

2. Luckasson R, Reeve A. Naming, defining, and classifying in mental retardation. Ment Retard. 2001;39(1):47-52.

3. Shevell M. Global developmental delay and mental retardation or intellectual disability: conceptualization, evaluation, and etiology. Ped Clinics North Am. 2008;55(5):1071-84.

4. Ellison JW, Rosenfeld JA, Shaffer LG. Genetic basis of intellectual disability. Annu Rev Med. 2012;64:441-50.

5. Manning M, Hudgins L. Professional Practice and Recommendations Committee. Array-based technology and recommendations for utilization in medical genetics practice for detection of chromosomal abnormalities. Genet Med. 2010;12 (11):742-5.

6. de Ligt J, Willemsen MH, van Bon BW, et al. Diagnostic exome sequencing in persons with severe intellectual disability. N Engl J Med. 2012;367(20):1921-9.
7. van Karnebeek CD, Scheper FY, Abeling NG, et al. Etiology of mental retardation in children referred to a tertiary care center: a prospective study. Am J Ment Retard. 2005;110(4):253-67.

8. Makela NL, Birch P, Friedman JM, Marra CA. Parental perceived value of a diagnosis for intellectual disability (ID): a qualitative comparison of families with and without a diagnosis for their child's ID. Am J Med Genet. 2009;149A(11):2393-402.

9. van Karnebeek CD, Stockler S. Treatable inborn errors of metabolism causing intellectual disability: a systematic literature review. Mol Genet Metab. 2012;105(3):368-81

10. van Karnebeek CD, Houben RF, Lafek M, Giannasi W, Stockler S. The treatable intellectual disability APP www.treatable-id.org: a digital tool to enhance diagnosis \& care for rare diseases. Orphanet J Rare Dis. 2012;7:47.

11. Michelson DJ, Shevell MI, Sherr EH, Moeschler JB, Gropman AL, Ashwal S. Evidence report: genetic and metabolic testing on children with global developmental delay: report of the Quality Standards Subcommittee of the American Academy of Neurology and the Practice Committee of the Child Neurology Society. Neurology. 2011;77(17):1629-35.

12. Battaglia A, Carey JC. Diagnostic evaluation of developmental delay/mental retardation: an overview. Am J Med Genet C. 2003; 117C(1):3-14.

13. Traynor BJ, Alexander M, Corr B, Frost E, Hardiman O. Effect of a multidisciplinary amyotrophic lateral sclerosis (ALS) clinic on ALS survival: a population based study, 1996-2000. J Neurol Neurosurg Psych. 2003;74(9):1258-61.

14. Williams J, Sharp GB, Griebel ML, et al. Outcome findings from a multidisciplinary clinic for children with epilepsy. Child Health Care. 1995;24(4):235-44.

15. Skotko BG, Davidson EJ, Weintraub GS. Contributions of a specialty clinic for children and adolescents with Down syndrome. Am J Med Genet A. 2013;161A(3):430-7.

16. Hanna MG, Wood NW. Running a neurogenetic clinic. J Neurol Neurosurg Psychiatry. 2002;73 Suppl 2:II2-4.

17. Engbers HM, Berger R, van Hasselt P, et al. Yield of additional metabolic studies in neurodevelopmental disorders. Ann Neurol. 2008;64(2):212-17.

18. Rauch A, Hoyer J, Guth S, et al. Diagnostic yield of various genetic approaches in patients with unexplained developmental delay or mental retardation. Am J Med Genet A. 2006;140(19):2063-74.

19. Guo L, Li BX, Deng M, et al. Etiological analysis of neurodevelopmental disabilities: single-center eight-year clinical experience in south China. J Biomed Biotechnol. 2011; ID318616:1-11.

20. Jauhari P, Boggula R, Bhave A, et al. Aetiology of intellectual disability in paediatric outpatients in Northern India. Dev Med Child Neurol. 2011;53(2):167-72.

21. Gahl WA, Markello TC, Toro C, et al. The National Institutes of Health Undiagnosed Diseases Program: insights into rare diseases. Genet Med. 2012;14(1):51-9.

22. Mackenzie A, Boycott KM. The future is now for rare genetic diseases. CMAJ. 2012; 184(14): 1603.

23. Bamshad MJ, Shendure JA, Valle D, et al. The Centers for Mendelian Genomics: a new large-scale initiative to identify the genes underlying rare Mendelian conditions. Am J Med Genet A. 2012;158A(7):1523-5. 


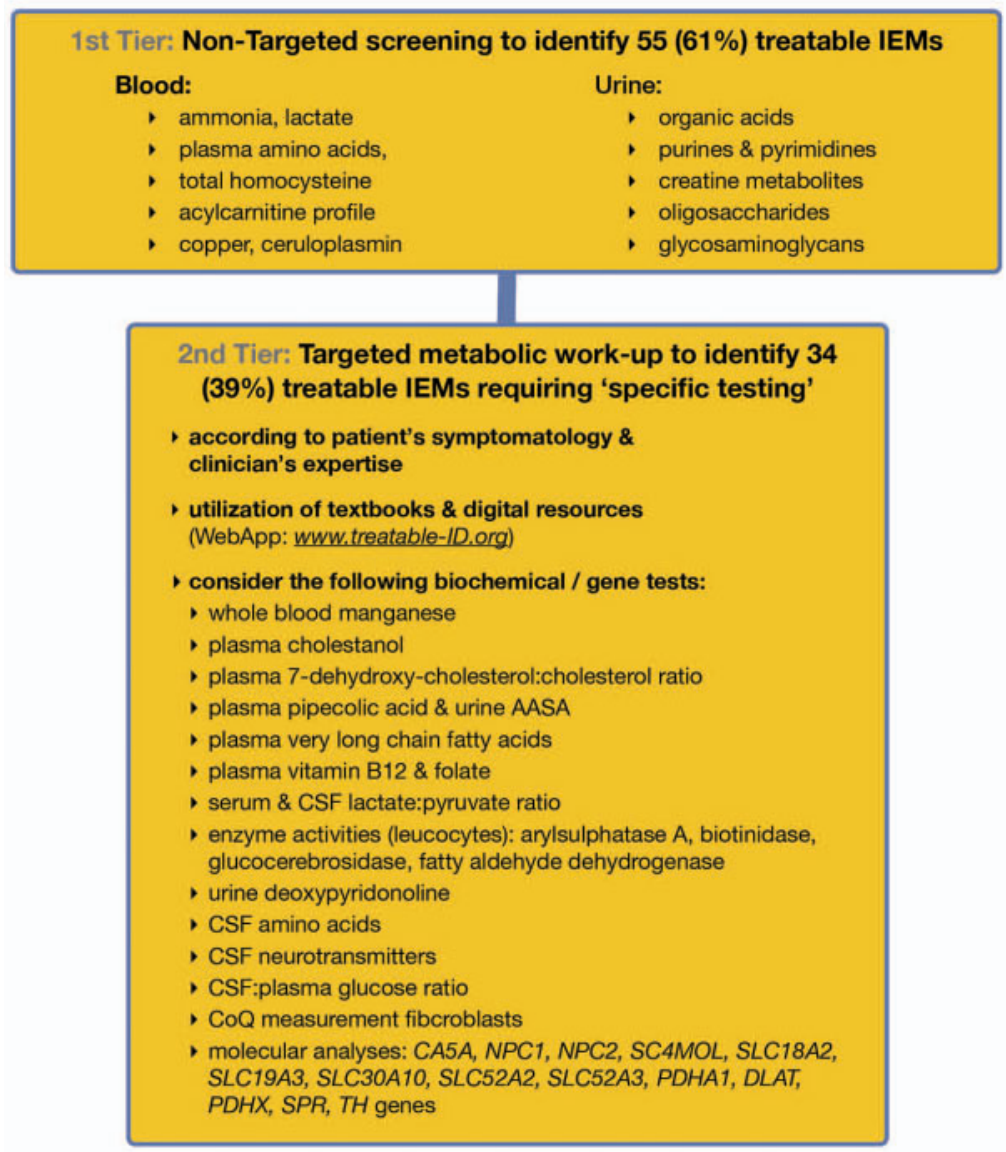

Supplementary Figure: The TIDE protocol, implemented in British Columbia Children's Hospital as 2-tiered algorithm to identify 81 treatable IEMs in ID patients.

\section{Supplementary Methods}

\section{Cost Calculation \& Comparison}

To assess the costs of the diagnostic work-up prior to and during the clinic, both to the health care system and to the families, the following data analyses and cost calculations were performed:

a) costs of diagnostic evaluation prior to $\mathrm{CDC}$ : laboratory testing (biochemical genetics, molecular genetics and cytogenetics), clinical diagnostic procedures (neuroimaging, biopsies, lumbar punctures), hospital admissions, and specialist consultations performed with the aim of determining the etiologic diagnosis. Complete chart reviews were performed; activities for disease monitoring or therapeutic purposes were excluded. The B.C. Medical Services Commission, the BCCH. Molecular Genetics and Biochemical Genetics Laboratory payment schedules were used to determine costs of consultations and laboratory/molecular genetic tests.

b) costs incurred by families prior to CDC: the average single person income in B.C. (Statistics Canada) was used to calculate the cost of one parent to take time off work in order to come to BCCH for an outpatient consultation. For families living less than $25 \mathrm{~km}$ away from B.C.C.H., a half day was calculated and for families living farther than $25 \mathrm{~km}$ from $\mathrm{BCCH}$ a full day. Travel costs were calculated by multiplying the number of visits to $\mathrm{BCCH}$ by the round trip distance at the Canada Revenue Agency accepted reimbursement rate of $\$ 0.53 / \mathrm{km}$.

c) CDC operating \& diagnostic costs: including coordination, consultations for each of the referring and evaluating physician, costs of laboratory tests ordered during the $\mathrm{CDC}$, and the average physician time to execute and follow-up on these.

Subsequently the costs incurred prior to the CDC were compared to the expenses that were incurred during the CDC. The intent was to provide an overview of both and identify specific areas for which the CDC improved cost-efficiency.

\section{Qualitative Evaluation \& Quality Improvement}

An evaluation framework was developed and executed to assess the CDC experience both for families and medical specialists. To provide a baseline on previous experiences and 
CDC expectations, as well as an opportunity for families to ask any additional questions prior to the clinic, the Evaluator interviewed parents per telephone. After the clinic, families and providers were sent a link to an online survey asking for their feedback of the clinic.

Family Experience. Following the patient's participation at the TIDE-CDC, patients/families were asked to complete an online survey about their satisfaction with the clinic. The survey comprised open and closed ended questions asking for input about their experience with the TIDE-CDC, including satisfaction with the degree to which family centered care was realized and multidisciplinary collaboration between specialist physicians supported their overall experience. Validated questions from the Measure of Processes of Care (MPOC-50) asked about patient/family perceptions surrounding the provision of general information about the clinic, specific information about the child's condition, enabling respectful and supportive care and enabling comprehensive and coordinated care were used to supplement the survey designed by the evaluator ${ }^{1}$. Survey questions used 10-point satisfaction rating scales as well as descriptive rating scales, which were numerically coded using Excel for analysis. Statistical software was not deemed necessary given the small patient number.

The survey software used was FluidSurveys. Anonymous data for each completed survey was stored in a database hosted on FluidSurvey's secure server in Ottawa. Password protection was put in place to ensure only authorized users could access the data from FluidSurveys' server.

The survey questions used to assess the impact and effectiveness of the Clinic were analyzed using descriptive statistics. Open-ended comments were analyzed via thematic analysis and used to compare experiences from pre to post TIDE$\mathrm{CDC}$ as well as to inform continuous improvements following the Plan-Do-Study-Act (PDSA) methodology by the TIDE-CDC team. To mitigate bias, all patients/families were asked to participate in the survey, and they were asked to participate prior to the clinic appointment. As well, neutral and non-leading questions were asked through a survey designed and disseminated by an independent, non-clinical, evaluator. Finally, all responses were anonymous.

Clinician Experience. Following each clinic, all clinicians were asked to complete an online survey assessing the impact of participating in the multidisciplinary clinic. Open and closed ended questions were developed by the evaluator to determine how working in a multidisciplinary team affected their clinical practices and how information learned from colleagues from other disciplines affected their understandings of treatable intellectual disabilities, the diagnostic process, and treatment recommendations. The survey questions were also developed to understand how the clinic supported synergistic working relationships demonstrated through collaboration and communication. Survey questions used 10-point satisfaction rating scales as well as descriptive rating scales, which were numerically coded using Excel for analysis.

The survey software used for the clinician survey is also FluidSurveys. The survey questions were analyzed using descriptive statistics and open ended comments were analyzed via thematic analysis and used to compare experiences from pre to post TIDE-CDC as well as to inform continuous improvements following the PDSA methodology by the TIDECDC team.

To mitigate bias, all clinicians were asked to participate in the survey. As with the patient/family survey, neutral and nonleading questions were asked through a survey designed and disseminated by an independent, non clinical evaluator. Finally, all responses were anonymous.

Quality improvement. As part of TIDE-CDC's commitment to quality improvement, the PDSA model was utilized to continuously improve the clinic. The CDC management team met quarterly to review the operations of the clinic and seek ways to improve (i.e. development of a standard operating manual, stricter patient recruitment and selection, communication, coordination, points of contact with patients, rounds moderation and evaluation questionnaires and tools, duration of evaluation). Changes were documented by the Manager and implemented for subsequent clinics under clinical/administrative co-management direction.

\section{REFERENCES}

1. King S, Rosenbaum P, King G. The Measure of Processes of Care: A means to assess family-centred behaviours of health care providers. Hamilton: McMaster University, Neurodevelopmental Clinical Research Unit; 1995.

\section{SuPPLEMENTARY RESUlTS}

\section{Illustrative case vignettes}

Aside from the high diagnostic yield, the true value of the collaborative approach for establishing a diagnosis through integrated clinical and laboratory expertise of a group of specialists, is best illustrated by the following eight case vignettes:

\section{Diagnosis can still be made 'clinically' (patients \#22 and 24)}

At the age of five years, this girl was referred to the clinic with global developmental delay, absence of speech, happy and easily excitable demeanour, tremulous movements, congenital microcephaly, strabismus, large mouth and prominent mandible. The clinical geneticist suspected Angelman syndrome at 1.5 years-of-age and ordered the appropriate cytogenetic tests; deletion and imprinting abnormalities of chromosome 15q11-13 were ruled out. $U B E 3 A$ gene analysis was not performed due to lack of funding. Chromosome micro-array and $M e C P 2$ gene testing were normal as well. With further evolution of her phenotype over time, specialists agreed that even in the absence of molecular confirmation, Angelman syndrome is the confirmed clinical diagnosis in this patient. No further testing will be performed, thereby ending this girl's diagnostic odyssey and allowing for proper management according to Angelman syndrome guidelines. Furthermore, genetic counselling was adapted given recurrence risk in a next child for the same couple is as high as $50 \%$ in case of a $U B E 3 A$ mutation (versus $1 \%$ in case of uniparental disomy or a deletion in the proband).

Similarly in case \#22, Sotos syndrome was agreed upon as the etiologic diagnosis despite the absence of an advanced bone age on X-Ray. This nearly three and a half years old girl's facial 
features had also become more typical for Sotos syndrome over the years, with macrodolichocephaly, long and protrusive forehead, hypertelorism, down slanting palpebral fissures and pointy chin. Together with the overgrowth (weight and height at the $98 \%$ percentile for age), mild developmental delay, hypotonia and temper tantrums, the specialists agreed that this diagnosis was clinically confirmed; repeat chromosome microarray (at higher resolution), and both NSDI (pending) and EZH2 gene (negative) sequencing were ordered as the only tests (provided on a research basis), and the patient will be managed accordingly.

\section{Unrecognized presentation of a known treatable disease (patient} \#6)

This two year-old had been extensively evaluated for (genetic) gastro-enterologic conditions by several specialists because of his global developmental delay, short stature, mild dysmorphisms, hepatomegaly, elevated transaminases and cholesterol, as well as diarrhea. During the clinic rounds, specialists agreed that his facial features, on the one hand, resembled those of his father, but on the other impressed as a 'doll face'; only then was the possibility raised of hepatic glycogen storage disease despite the absence of documented hypoglycaemia. The patient was further investigated with normoglycemia on prolonged fasting test; however, a gene panel did confirm X-linked Glycogen Storage Disease Type IXa due to a pathogenic mutation in the PHKA2 gene. Since the appropriate treatment has been initiated, with frequent meals and snacks as well as cornstarch overnight to avoid hypoglycaemia, this boy's somatic and psychomotor development has improved.

\section{Re-interpretation of seemingly innocent chromosomal copy number variants (CNVs) (patient \#8)}

This 13-year-old boy had been extensively evaluated for microcephaly, dysmorphic features, expressive language and social skills delay, coordination deficit, ADHD and mood liability, sleep disturbances, history of watery diarrhea, growth retardation, and alopecia areata. Chromosome micro-array analysis had been performed two years previous to the CDC visit, revealing a maternally inherited deletion of chromosome $15 q 26.1$ region. At the time this was a previously unreported variant of unknown significance, thus concluded to be benign in view of its presence in the unaffected mother. However, upon reevaluation of the most recent literature, previous testing with normal results, as well as the patient's clinical features, the multi-disciplinary CDC team concluded this CNV causal of the phenotype. The alopecia areata was considered an unrelated finding by the dermatologist, affecting $2 \%$ of the general population and treated according to international protocols. This case demonstrates the importance of re-evaluating older test results of which the significance may change according to increasing knowledge; certainly in the field of genetics, no single physician can keep completely up to date. In this multidisciplinary setting it was possible to confirm this chromosomal abnormality as etiologic as it was unanimously agreed upon that other diagnoses had been ruled out, and that the alopecia was an unrelated finding.
Multifactorial etiology with a potentially treatable component (patient \#9)

For this four-year old girl the most evident etiology, teratogenicity, was found to be an incomplete explanation for her multiple congenital anomalies with recurrent fractures. After intra-uterine exposure to high dose methotrexate ingested by mother for rheumatoid arthritis and unaware of pregnancy, this girl was born with the following anomalies: coronal synostosis, facial dysmorphisms, left pulmonary artery sling, tracheal diverticulum, tracheomalacia, short stature and mild rhizomelic shortening of the limbs. She is G-tube fed and ventilator dependent and admitted to hospital for severe airway infections. She suffers recurrent pathologic fractures with diffuse decreased mineralization (translucency on radiographs), and was extensively evaluated by diverse specialists; renal and endocrine causes were ruled out.

Metabolic diseases service ruled out hypophosphatasia and as incidental finding confirmed the homozygous CPT1a (P479L) variant in her and advised regular intake to avoid hypoglycaemia. Re-examined in the clinic, bluish sclerae and translucent skin (normal enamel and nails) were identified suggestive of a connective tissue disorder. A skin biopsy was performed for collagen studies, revealing $50 \%$ reduction of type I pro-collagen and collagen synthesis and secretion. COL1A and COL1A2 molecular analysis did not reveal any mutations. This patient was enrolled into the TIDEX study for whole exome sequencing and likely causal variants identified in the DSEL gene (under validation) were identified. If confirmed pathologic, these results will decide further management. Most importantly, the insight of a multi-factorial etiology in this girl (i.e. that the pathologic factures did not fit the MTX embryopathy) is significant for the family in that mother can rid herself of the heavy feelings of guilt surrounding the teratogen exposure during the period when the pregnancy was unknown to her.

\section{Diagnosis per exclusionem (patient \#4)}

Multi-specialist consensus facilitates the process of confirming a diagnosis "per exclusionem". Illustrative for this is patient \#4, a ten year old girl presenting with punctuate basal ganglia calcifications, constipation / diarrhea, familial ADHD with tics, and a maternally inherited chromosomal Xp22.31 duplication. Extensive testing had been performed to rule out infectious, metabolic, teratogenic causes of the intracerebral abnormalities. In view of the benign medical history and good developmental progress of this child, combined with the normal results of complete and extensive testing in the past, it was decided by the clinic team that benign familial basalganglia calcifications is the diagnosis per exclusionem, and that the copy number variation predisposed her to a learning disorder. The parents were satisfied with this explanation and no further testing was initiated.

\section{“We still don't know; let's wait and see” (patient \#16)}

The decision to stop diagnostic testing in a child with a "wait and see' approach, is often hard to make as the array of possible diagnoses and investigations in ID/GDD is endless, and so this is often seen as 'giving up'. However this can benefit the patient tremendously as unnecessary tests and burden are avoided, and 
the family can adjust to the idea of not having a diagnosis, while in the meantime technology advances may allow success in the future. It is often easier for a group of specialists than one individual to make this well-informed decision. This scenario is illustrated by (amongst others) patient \#16, presenting with mild intellectual disability, hypotonia, joint hypermobility, bilateral inguinal hernia inguinales, pulmonary stenosis, dysmorphic features, scoliosis, easy bruising, and refractive amblyopia. The family history is remarkable; the father displays psychiatric disturbances, dyslexia, similar facial features and inguinal hernia and the maternal history includes muscle cramps and cardiac (valvular) anomalies. Features are suggestive of a connective tissue and/or muscle disorder and the pattern of inheritance cannot be clearly defined. The most likely etiology is polygenic or multifactorial. The group consensus comprised a list of conditions (congenital disorder glycosylation, metal disorder, Smith-Lemli-Opitz syndrome, Myotonic Dystrophy, chromosomal copy number variant) to rule out, and if all were negative to stop testing and follow-up in two to three years by the referring clinical geneticist.

Platform for next generation sequencing and gene discovery (patient \#11)

The patient is a six-year old boy with congenital progressive microcephaly, supra-tentorial brain atrophy, progressive demyelination, visual impairment, profound intellectual disability without psychomotor development since birth and a severe seizure disorder. The 'million dollar' diagnostic work-up including involvement of expert neuroradiologists in the Netherlands did not reveal a diagnosis in this patient. During the clinic, specialists agreed to select this non-consanguineous patient for our TIDEX study and whole exome sequencing (WES) was performed on the trio, revealing compound heterozygosity for mutations in the DSCAML1 gene. The Dscam-Like1 cell adhesion molecule has been tied to neuronal arborisation and to the formation of synapses. Further validation studies are currently underway, including a 3T-MRI scan and sophisticated retinal imaging. This discovery has major impact on pathophysiologic understanding of congenital brain abnormalities as well as potential for research collaborations. Although not a causally treatable disease, implications of this diagnosis are considerable for the family who wishes for another child and can be provided with appropriate prenatal guidance to avoid recurrence, as well as closure and relief of guilt.

Overall, a total of seven cases have been selected in the CDC for WES. MSP funding to have this done via a commercial company on a clinical basis has only been obtained for one patient (\#12), based on her severe clinical situation with lifelong admission in hospital for respiratory insufficiency, severe hypotonia and possible cranial nerve palsy, global developmental delay, dysautonomia, severe hypotonia, and the concomitant implications of a genetic diagnosis for prognosis and management. Variants in the Crisponi syndrome gene (CRLF1) were identified and validated. For six other patients with a specific phenotype suggestive of a Mendelian disorder and with potential for discovery and target for treatment, WES has been performed on a research basis, via the TIDEX study (patients \# 2, 3 9, 10, 11, 23) with data processed in the bioinformatics pipeline, a candidate gene hypothesis formulated and a translational clinical research team ready to interpret and validate the results. 